Results Our LCA model estimated the prevalence of treated asthma in Wales in 2011-2012 as 8.9\% (95\% CI: 8.7\%-9.1\%), which was higher than estimates from the Quality and Outcome Framework (6.9\%), but lower than both the prevalence of selfreported treated asthma estimated by the Welsh Health Surveys in $2011(11.0 \%)$ and $2012(10.0 \%)$ and the prevalence of 'GP reported and treated asthma' from the 'True Costs of Asthma in the UK' project (13.0\%). In our model, prescription of any asthma medication had the highest accuracy among other observed variables (sens. $=99 \%$; spec. $=\mathrm{PPV}=\mathrm{NPV}=$ $100 \%$ ), while asthma diagnosis variable had a lower accuracy (sens. $=66 \%$; spec. $=94 \% ; \mathrm{PPV}=51 \% ; \mathrm{NPV}=97 \%)$. In the same sample, COPD prevalence was $2.0 \%$ (95\% CI: $1.9 \%$ $2.1 \%$ ) with only $2.8 \%$ of those classified as asthmatics were also classified as having COPD.

Conclusion Our LCA model provides a reasonable, data-driven, reference identification of people with treated asthma in Wales. Further work is needed to explore potential reasons for the observed differences in the estimates from other sources.

\section{P226 IMPAIRED RESPIRATORY HEALTH STATUS IN THE UK HIV INFECTED POPULATION DESPITE THE USE OF ANTIRETROVIRAL THERAPY}

${ }^{1}$ JP Brown, ${ }^{2} \mathrm{~J}$ McGowan, ${ }^{1} \mathrm{H}$ Chouial, ${ }^{3} \mathrm{~S}$ Capocci, ${ }^{2} \mathrm{C}$ Smith, ${ }^{1} \mathrm{D}$ Ivens, ${ }^{2} \mathrm{~F}$ Lampe, ${ }^{1} \mathrm{M}$ Johnson, ${ }^{1} \mathrm{~L}$ Sathia, ${ }^{2} \mathrm{~A}$ Rodger, ${ }^{1} \mathrm{M}$ Lipman. ${ }^{1}$ Departments of Respiratory and HIV Medicine, Royal Free London NHS Trust, London, UK; ${ }^{2}$ Department of Infection and Population Health, University College London, London, UK; ${ }^{3}$ Division of Medicine, University College London, London, UK

\subsection{6/thoraxjn-2015-207770.362}

Background The widespread use of antiretroviral therapy (ART) has led to a reduction in HIV related opportunistic infections. An increase in chronic non-HIV related co-morbidities has been observed in stable HIV positive individuals receiving ART. The extent to which HIV infection remains an independent risk factor for respiratory disease despite the use of antiretroviral therapy is uncertain and few studies have systematically evaluated respiratory disease in HIV-infected populations with access to antiretroviral therapy.

Aims We sought to evaluate the frequency of (a) smoking and (b) respiratory symptoms and (c) spirometric impairment in the ambulatory UK adult HIV infected population, compared to HIV uninfected controls.

Methods HIV-positive participants were recruited from a large HIV care service, HIV uninfected participants were recruited from Sexual Health services (where recruitment was stratified by age to approximate that of the HIV positive subjects). Participants completed a questionnaire which included questions on smoking history and respiratory health status using the St George's Respiratory Questionnaire (SGRQ), and undertook spirometry without bronchodilation.

Results 249 participants were recruited between April and July 2015 (Table 1). $28 \%$ of HIV positive and 33\% of HIV negative participants were current smokers $(\mathrm{p}=0.22)$. 9\% of HIV positive and $7 \%$ of HIV negative participants had an $\mathrm{FEV}_{1} / \mathrm{FVC}$ of $<0.7$ ( $\mathrm{p}=0.38$ ). $92 \%$ of HIV positive participants were using antiretroviral therapy, $86 \%$ had an undetectable plasma HIV viral load and mean CD4 count was 684 cells $/ \mu \mathrm{L}$.

\begin{tabular}{|c|c|c|c|}
\hline & $\begin{array}{l}\text { HIV positive } \\
(\mathrm{N}=181)\end{array}$ & $\begin{array}{l}\text { HIV negative } \\
(\mathrm{N}=68)\end{array}$ & \\
\hline Age [years] & $50(43-56)$ & $44(38-52)$ & $P=0.006$ \\
\hline Using antiretroviral therapy & $92 \%$ & & \\
\hline CD4 count [cells/ $\mu \mathrm{L}]$ & $617(458-839)$ & & \\
\hline Male & $79 \%$ & $68 \%$ & $P=0.065$ \\
\hline Current smoker & $28 \%$ & $33 \%$ & $P=0.22$ \\
\hline $\mathrm{FEV}_{1}$ & $3.43(0.86)^{*}$ & $3.20(0.78)^{*}$ & $P=0.08$ \\
\hline FVC & $4.24(1.06)^{*}$ & $3.87(0.98)^{*}$ & $P=0.02$ \\
\hline $\mathrm{FEV} 1 / \mathrm{FVC}<0.7$ & $9 \%$ & $7 \%$ & $P=0.55$ \\
\hline Total score & $12(6-29)$ & $8(3-18)$ & $P=0.032$ \\
\hline
\end{tabular}

Significantly higher SGRQ scores were observed in HIV positive participants than HIV-negative participants with a median total SGRQ score of 12 for those with HIV infection and 8 for the HIV negative participants $(\mathrm{p}=0.03)$. In a linear regression (log scale) model, HIV infection was associated with a $62 \%$ increase $(95 \%$ CI 1.19-2.21, p < 0.01) in SGRQ in unadjusted analysis and $48 \%$ increase $(1.08-2.02, \mathrm{p}=0.01)$ in a multivariable analysis adjusting for age, gender and smoking status.

Conclusions Despite widespread use of ART, HIV infection is independently associated with impaired respiratory health status. This does not appear to result from current smoking or obstructive lung disease.

\section{P227 LUNG CANCER DIAGNOSIS AT EMERGENCY ADMISSION - HOW DOES DORSET COMPARE?}

${ }^{1} \mathrm{C}$ Bradley, ${ }^{2} \mathrm{E}$ Harvey, ${ }^{3} \mathrm{~N}$ Ranaweera. ${ }^{1}$ Royal Hampshire County Hospital, Winchester, UK; ${ }^{2}$ Poole General Hospital, Poole, UK; ${ }^{3}$ Royal Bournemouth Hospital, Bournemouth, UK

\subsection{6/thoraxjnl-2015-207770.363}

Introduction Survival for lung cancer patients in the UK is worse than in comparable countries, at least partly because they present with more advanced disease. ${ }^{1}$ Recent data suggest that rural residence is associated with an increased risk of early death in lung cancer. $^{2}$

As our region encompasses rural areas, we investigated rates of emergency admission at the three major hospitals in our region and factors which may lead to this.

Methods We retrospectively identified new presentations of lung cancer as emergencies from August to October 2014. We gathered patient demographics, mortality and GP presentation data and compared them with local lung cancer database data for the same time period.

Results We identified 41 new lung cancer diagnoses in this period, from a total of 119 new diagnoses. This gives an emergency diagnosis rate of $34.5 \%$, comparable to national figures of $39 \%{ }^{1}$ However, there was significant variation $(21-43 \%)$ between the three sites.

When analysed by gender, only $30 \%$ of male diagnoses were made at emergency presentation, compared with $41 \%$ of females. Unfortunately our sample size was not large enough to demonstrate statistical significance $(\mathrm{p}=0.22)$.

GP data were available for 28 patients, of whom 17 had reported symptoms to their GP. The median duration between 
first reporting symptoms to the GP and being admitted as an emergency was only 4 days.

As expected, staging in emergency patients was significantly higher than in those diagnosed as outpatients (Figure 1, $\square$ indicates $\mathrm{p}<0.001)$. Mortality at 3 months was comparable: $56 \%$ compared with $13 \%(\mathrm{p}<0.001)$.

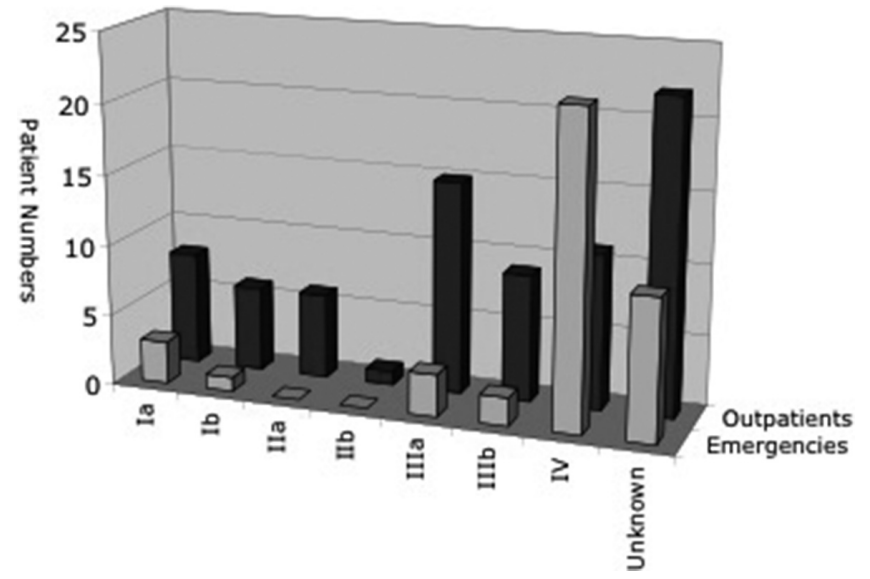

\section{Abstract P227 Figure 1}

Conclusions Emergency presentation data for our region are comparable with national figures, with comparable mortality.

Further examination of variability in admission rates between our hospitals, and possible gender inequality, could suggest future avenues to improve early lung cancer diagnosis.

\section{REFERENCES}

1 Walters S, Maringe C, Coleman MP, et al. Lung cancer survival and stage at diagnosis in Australia, Canada, Denmark, Norway, Sweden and the UK: a population-based study 2004-2007. Thorax 2013;68:551-564

2 O'Dowd EL, McKeever TM, Baldwin DR, et al. What characteristics of primary care and patients are associated with early death in patients with lung cancer in the UK? Thorax 2015;70:161-168

\section{Improving patient care in cystic fibrosis}

\section{P228 IMPACT OF SOCIAL MEDIA ON ADULT CF CENTRES ACROSS THE UK}

G Fitch, C Etherington, P Whitaker, D Peckham. Leeds Adult CF Unit, Leeds Teaching Hospitals NHS Trust, Leeds, UK

\subsection{6/thoraxjnl-2015-207770.364}

Background People with chronic health conditions use social media to share experiences and seek advice from peers. Similarly healthcare organisations are using these tools to engage and communicate with patients. The aim of this study was to investigate the opinions of health care professionals on the use, motives and impact of social media used by patients and staff in UK CF centres. Methods Members of the Leeds adult CF MDT developed an online survey with 18 core questions relating to social media. The survey was sent to the clinical lead of each CF centre in the UK. The lead was asked to complete the survey and to pass it on to key members of their team to complete. Mixed methods were used with categorical, Likert and free text options.
Results 66 respondents from 9 professional groups and 19 centres across the UK completed the questionnaire. 16/44 professionals had never used social media and 35/60 reported that their centre had no social media presence. There was belief that social media was having a negative impact on certain aspects of care including spreading inappropriate gossip and in some cases bullying. Only $30.2 \%$ of respondents felt social media had a positive impact at their centre in contrast to $73.3 \%$ of responders who felt social media had been used in a negative way. Verbal communication, directing individuals to use departmental social media channels and communication of acceptable behaviour to all patients via a website or newsletter were methods shared to deter negative content. Despite their negative experiences many felt social media had the potential to be a positive experience in areas such as communication with patients, education, adherence, peer support, amongst others.

Conclusion Negative experiences involving social media use by patients predominate. Different methods to tackle these problems have been adopted. Sharing our experiences and being alert to such problems should help minimise distress in the future. Many potential areas of positive use exist and should be exploited.

\section{P229 USE OF INHALED ANTIBIOTICS IN CF BURKHOLDERIA SPP CHRONIC INFECTION}

JE Marlow, K Delisle, S Ajab, M Ledson, J Greenwood, M Walshaw. Liverpool Heart and Chest Hospital, Liverpool, UK

\subsection{6/thoraxjnl-2015-207770.365}

Introduction Although CF guidelines recommend that patients chronically infected with Pseudomonas aeruginosa should be offered inhaled antibiotics to help maintain pulmonary function and reduce exacerbation frequency, there is no such advice for those infected with Burkholderia spp, some of which confer a much worse prognosis.

Methods To look at the potential for this, we reviewed the microbiology of all adults attending our large unit (300 patients), paying attention to those with chronic Burkholderia infection, any co-existing pathogens, antibiotic sensitivity patterns, and the use of inhaled antibiotics.

Results Twenty two patients (7.3\%) are infected with Burkholderia spp (6 multivorans, 8 cenocepacia, 1 genomovar IIIa cable pilus negative, 3 genomovar IIIb, 3 vietnamensis, and 1 dolosa. For sensitivity patterns (defined as $>10 \mathrm{~mm}$ inhibition by disc diffusion) see Table 1.9 patients (41\%) are co-infected (4 with Pseudomonas aeruginosa, 3 Staph aureus, 2 MRSA, 1 Stenotrophomonas maltophilia).

\begin{tabular}{lllllll}
$\begin{array}{l}\text { Abstract P229 Table } 1 \\
\text { Burkholderia } \\
\text { spp }\end{array}$ & Multivorans & \multicolumn{5}{c}{ Sensitivity patterns of Burkholderia spp } \\
\hline Colomycin & $0 / 6$ & $0 / 8$ & $0 / 1$ & $0 / 3$ & $0 / 3$ & $0 / 1$ \\
Ceftazidime & $2 / 6$ & $4 / 8$ & $1 / 1$ & $2 / 3$ & $3 / 3$ & $1 / 1$ \\
Aztreonam & $2 / 6$ & $0 / 8$ & $1 / 1$ & $2 / 3$ & $3 / 3$ & $1 / 1$ \\
Tobramycin & $0 / 6$ & $4 / 8$ & $0 / 1$ & $0 / 3$ & $2 / 3$ & $0 / 1$ \\
Amikacin & $1 / 6$ & $0 / 8$ & $0 / 1$ & $0 / 3$ & $3 / 3$ & $1 / 1$ \\
Ciproxin & $1 / 6$ & $0 / 8$ & $0 / 1$ & $0 / 3$ & $0 / 3$ & $0 / 1$ \\
\hline
\end{tabular}

